\title{
Kynurenine Pathway Metabolites as Biomarkers in Alzheimer's Disease
}

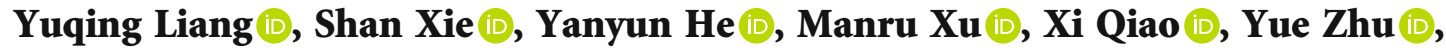 \\ and Wenbin $\mathrm{Wu}$ (iD
}

Department of Geriatrics, Hospital of Chengdu University of Traditional Chinese Medicine, Chengdu, Sichuan 610072, China

Correspondence should be addressed to Wenbin Wu; wwb1201@vip.sina.com

Received 10 October 2021; Revised 21 December 2021; Accepted 31 December 2021; Published 19 January 2022

Academic Editor: Jinming Han

Copyright $\odot 2022$ Yuqing Liang et al. This is an open access article distributed under the Creative Commons Attribution License, which permits unrestricted use, distribution, and reproduction in any medium, provided the original work is properly cited.

\begin{abstract}
Alzheimer's disease $(\mathrm{AD})$ is a progressive neurodegenerative disorder that deteriorates cognitive function. Patients with AD generally exhibit neuroinflammation, elevated beta-amyloid $(\mathrm{A} \beta)$, tau phosphorylation ( $p$-tau), and other pathological changes in the brain. The kynurenine pathway (KP) and several of its metabolites, especially quinolinic acid (QA), are considered to be involved in the neuropathogenesis of $\mathrm{AD}$. The important metabolites and key enzymes show significant importance in neuroinflammation and AD. Meanwhile, the discovery of changed levels of KP metabolites in patients with AD suggests that KP metabolites may have a prominent role in the pathogenesis of AD. Further, some KP metabolites exhibit other effects on the brain, such as oxidative stress regulation and neurotoxicity. Both analogs of the neuroprotective and antineuroinflammation metabolites and small molecule enzyme inhibitors preventing the formation of neurotoxic and neuroinflammation compounds may have potential therapeutic significance. This review focused on the KP metabolites through the relationship of neuroinflammation in $\mathrm{AD}$, significant $\mathrm{KP}$ metabolites, and associated molecular mechanisms as well as the utility of these metabolites as biomarkers and therapeutic targets for $\mathrm{AD}$. The objective is to provide references to find biomarkers and therapeutic targets for patients with $\mathrm{AD}$.
\end{abstract}

\section{Introduction}

Over 55 million people globally live with dementia, but only $25 \%$ are properly diagnosed since diagnostic criteria for dementia are unclear [1]. Alzheimer's disease $(\mathrm{AD})$ is the main cause of dementia. The primary clinical manifestation of $\mathrm{AD}$ is cognitive impairment, characterized by a comprehensive decline in cognitive, behavioral, language, and other brain functions. Pathological manifestations of $\mathrm{AD}$ involve the extracellular deposition of beta-amyloid $(\mathrm{A} \beta)$, intracellular neurofibrillary tangles (NFT), tau phosphorylation ( $p$ tau), and so on. Currently, the pathogenesis of $\mathrm{AD}$ remains unknown and many hypotheses are based on observed pathological changes (e.g., the $\mathrm{A} \beta$ waterfall hypothesis) $[2,3]$. The discovery of inflammatory biomarkers, interleukin(IL-) $1 \beta[4,5]$, and $\mathrm{AD}$ risk genes associated with innate immune function, apolipoprotein $\mathrm{E}$ (APOE) and triggering receptor expressed on myeloid cells 2 (TREM2), in $\mathrm{AD}$ patients suggests that neuroinflammation is essential in pathogenesis $[2,6]$.

The kynurenine pathway (KP) is crucial for peripheral and central catabolism of the essential amino acid, Ltryptophan (L-TRP). An imbalance in KP metabolism is a significant contributor to neuroinflammation. For example, overactivation of KP may result in excessive production of the excitotoxin quinolinic acid (QA) by activated microglia and perivascular and infiltrating macrophages [7-9]. A low level of cinnabarinic acid (CA) likely protects neurons but can be oxidized by 3-hydroxyanthranilic acid (3-HANA) to produce free radical superoxide anion and hydrogen peroxide $[10,11]$. Conversely, picolinic acid (PA) has a dual function in immune regulation and can limit the excitatory toxicity of QA [12]. An interaction exists between KP metabolite imbalance and neuroinflammation. In a casecontrol study of clinical and biomarker-confirmed $\mathrm{AD}$ patients and cognitively healthy controls with paired plasma 
and cerebrospinal fluid (CSF) samples, metabolic profiling has been performed and the results showed that the specific association of amino acids and L-TRP catabolites with AD CSF biomarkers suggests a close relationship with core $A D$ pathology [13]. And another metabolomic analysis of AD patients and healthy seniors suggests that the involvement of polyamine and L-TRP-KYN metabolisms was observed in the postmortem cerebrospinal fluid (PCSF) samples [14]. In addition, studies found that KP metabolites are associated with pathological changes and cognitive function in $\mathrm{AD}$, such as QA [7-9, 15-25]. This work first discusses the mechanisms of $\mathrm{AD}$ neuroinflammation and $\mathrm{KP}$ regulation. Second, we summarized the relationship between neuroinflammation in $\mathrm{AD}$ and significant $\mathrm{KP}$ metabolites and molecular mechanisms. Ultimately, we discuss the applicability of KP metabolites as biomarkers for $\mathrm{AD}$ and provide references for the search for biomarkers and therapeutic targets.

\section{Neuroinflammation in AD}

Neuroinflammation is a response within the central nervous system caused by pathological damage, such as infection, trauma, ischemia, and toxin accumulation. This process involves the production of large quantities of proinflammatory cytokines, such as IL- $1 \beta$ and IL- 6 and tumor necrosis factor- (TNF-) $\alpha$; chemokine ligands, such as chemokine (C-C motif) ligand (CCL) 1 and CCL5; and small molecule messengers, such as prostaglandin, nitric oxide (NO), and reactive oxygen species (ROS) [26]. These mediators are produced by resident central nervous system (CNS) glia (microglia and astrocytes), endothelial cells, and peripherally derived immune cells [26-28]. Neuroinflammation is a critical pathophysiological feature of $\mathrm{AD}$ and may be a cause of the condition [29].

Microglial surface receptors recognize pathogen and paraprotein (e.g., $\mathrm{A} \beta$ ) and induce microglial activation through endocytosis. Chemokine receptors and interferons are activated. These latter molecules are major components of the neuroinflammatory process [30,31]. Reactive microglia are closely colocated with amyloid plaques in $\mathrm{AD}$ patients. These cells exhibit diverse phenotypes and interact with $\mathrm{A} \beta$ and tau types and with neuronal circuits $[29,32]$. Microglia activated by $\mathrm{A} \beta$ produce the proinflammatory cytokines (such as IL-1 $\beta$, IL-6, IL-8, and TNF), antiinflammatory cytokine (transforming growth factor- (TGF) $\beta$ ), chemokines (such as monocyte chemotactic protein-1 (MCP-1) and macrophage inflammatory protein-1 $\alpha$ (MIP$1 \alpha)$ ), cell adhesion molecules, NO, and ROS that lead to neurological dysfunction and death [33-36]. And the microglia receptors mainly include toll-like receptors (TLR), such as TLR1 and TLR2, and CD14, CD47, $\alpha 6 \beta 1$ integrin, and scavenger receptors. In turn, these receptors activate molecular pathways that induce microglial phenotypic changes [37-39]. For instance, TLR2 is a primary receptor for $A \beta$ peptide to trigger neuroinflammatory activation, which can recognize and bind to A $\beta$ and induce IL- 8 and TNF expression [39]. The transition to disease-associated microglia is associated with downregulation of homeostatic genes and upregulation of genes with recognized association with $\mathrm{AD}$, including APOE, TREM2, and TYRO protein tyrosine kinase-binding protein (TYROBP) [40]. Microglial activation depends largely on TREM2, and the role of TREM2 is important in the pathogenesis of $\mathrm{AD}$ [40-42]. The TREM2-TYROBP pathway is the main microglial signaling pathway and has been found to interact with both $\mathrm{A} \beta 42$ and tau-induced pathways at the gene expression level [43]. Several studies demonstrated that TREM signaling could suppress half of the pathways induced by $\mathrm{A} \beta$, implying a defensive role for microglia, and in a tau-TREM2TYROBP coexpression model, microglia acted synergistically to exacerbate tau-mediated neurodegeneration [44].

Microglia in aged brains show functional impairment and are prone to chronic activation that may contribute to $\mathrm{AD}[45,46]$. Microglial activation is a key element in promoting neuroinflammation and is closely related to pathological changes of $\mathrm{AD}$ [29]. Further, the peak in microglial activation may occur during the preclinical phase of $\mathrm{AD}$, accompanied by $\mathrm{A} \beta$ deposition. $\mathrm{A} \beta$ clearance in later stages of AD decreases and tau accumulates. This condition compromises the defense function of microglia and triggers continuous and harmful microglial activation [29]. However, activation of microglia appears to inhibit progression in patients with mild cognitive impairment (MCI) [47]. These microglia may degrade and clear $\mathrm{A} \beta[30,31,33,48-50]$. Microglial responses to deferent pathological stimuli substantially alter $\mathrm{AD}$ progression. Effects mediated by microglia exhibit different effects along the disease trajectory depending on individual susceptibility and may be influenced by prior microglial priming [29].

Elevated proinflammatory cytokines are observed in the serum, brain, and CSF of AD patients [51, 52]. A significant correlation between levels of proinflammatory cytokines and cognitive decline is likely $[53,54]$. Several meta-analyses and systematic reviews have described this relationship and suggest that $\mathrm{AD}$ is accompanied by peripheral and CNS-derived inflammatory processes $[4,5]$. Zenaro et al. found that neutrophil infiltration in the CNS induces a neuroinflammatory cascade via LFA-1 integrin. This process exacerbates the pathological and cognitive decline in AD [55]. CXCL12 injection in vivo induces an increase in monocytes in the circulation of mice, and CXCL12 may promote migration of monocytes into the brain perivascular space and their transformation into PVMs. The latter action may be due to the upregulation of PECAM-1, which contributes to neuroinflammation and memory decline without crossing the blood-brain (BBB) or spinal cord barrier (SCB) [56]. Matrix metalloproteinases can aggravate inflammatory damage to the BBB by activating free radicals and proinflammatory cytokines [57-59]. Multiple microRNAs form positive and negative regulatory feedback loops with transcription factors, participate in neuroinflammatory responses caused by hypoxia/ischemia, and alter protein kinase activity and tau phosphorylation patterns in the brain via activating glial cells or affecting the expression of cytokines in neurons [60, 61]. Knocking out of Calhm2, a calcium homeostasis regulatory protein, in $\mathrm{AD}$ model mice significantly reduced inflammatory activation and increased the phagocytosis of 
microglia. $A \beta$ deposition, neuroinflammation levels, and cognitive impairment of mice subsequently improved. Thus, neuroinflammation processes are closely related to Calhm2 regulation of calcium influx in microglia [62].

Activation of the inflammasome in the CNS is the key to establishing a chronic inflammatory environment that leads to neuronal dysfunction [29]. The recombinant NLR family, pyrin domain-containing protein 3 (NLRP3) inflammasome, in particular, is composed of nod-like receptor family members NLRP3, apoptosis-associated speck-like protein containing a CARD (ASC), and procaspase-1. This protein complex has become an important focus of research due to its central role in neuroinflammatory pathways [63]. NLRP3 recognizes danger signals of bacteria, viruses, and other endogenous factors and activates caspase- 1 to induce the production of proinflammatory cytokines IL- $1 \beta$ and IL-18 $[63,64]$. Microglia are critical for the initial appearance of $\mathrm{A} \beta$ plaques and the structure of the NLRP 3 inflammasome. These cells complete the assembly of the NLRP3 inflammasome, leading to the formation of ASC that contributes to the deposition of plaques $[65,66]$. A high concentration of $\mathrm{A} \beta$ can induce the expression of IL- $1 \beta$ and TNF- $\alpha$ in glial cells; $\mathrm{A} \beta$ deposition can also promote tau lesions by activating the NLRP3 inflammasome. This activation can also induce phosphorylation of tau accompanied by upregulation of inflammatory factors, such as IL-1 $\beta$ and IL-19 $[64,67]$. A $\beta 1-42$ peptide activates the TLR2, TLR4, and TLR6 to promote neuroinflammation [39]. The process continues with immune system activation and the production of inflammatory mediators [68]. Levels of tau phosphorylation decrease compared with the normal mice after injecting $\mathrm{A} \beta$ into the head of NLRP3- or ASC-deficient mice [67]. An NLRP3 inhibitor, OLT1177, reduces the activation of microglia and the formation of $\mathrm{A} \beta$ plaques in the cerebral cortex in an APP/PS1 mouse model of AD. These mice also showed less cognitive impairment $[65,69]$.

Overall, a correlation exists between neuroinflammation and $\mathrm{AD}$; treatment for relevant markers of neuroinflammation can alleviate the progression of the disease.

\section{The Kynurenine Pathway}

L-TRP is an essential amino acid obtained from the diet. LTRP is a cross-kingdom starting material for critical downstream metabolites involved in the synthesis of some indole-related compounds, such as 5-hydroxytryptophan (5-HT, serotonin), melatonin (MT), and kynurenine (KYN) [70-73]. L-TRP exists in unbound and bound forms in the blood in a ratio of about 9 to 1 . A balance between these forms in the circulation is important even though a big difference exists in their relative concentrations. Only free L-TRP can be transported across the BBB [74].

The KP is central to peripheral and central catabolism of L-TRP (Figure 1). L-TRP concentrations in healthy adult human serum range from $1,000-50,000 \mathrm{ng} / \mathrm{mL}$. The concentration of KYN is generally only one-tenth that of L-TRP $[75,76]$. Only $2 \%$ of L-TRP is converted to $5-\mathrm{HT}$ and MT that are critical for regulation of sleep, mood, appetite, and metabolism [71, 77]. Oxidative cleavage of the indole ring of L-TRP is followed by formation of N-formyl-L-kynurenine (NFKYN) by indoleamine-2,3-dioxygenase (IDO1 and IDO2) and tryptophan 2,3-dioxygenase (TDO) [78-80]. NFKYN is promptly degraded to KYN by kynurenine formamidase or kynurenine formylase [81, 82]. NFKYN is metabolized to KYN, the first stable intermediate metabolite, by formamidase. KYN is the central metabolite of the KP and is catabolized to anthranilic acid (AA), 3-hydroxy-Lkynurenine (3-HK), and kynurenic acid (KYNA) by kynureninase (KYNU), kynurenine 3-monooxygenase (KMO), and kynurenine aminotransferase I-IV (KAT I-IV), respectively [81-83]. $3-\mathrm{HK}$ is converted to 3 -hydroxyanthranilic acid (3-HANA), the precursor to the neurotoxic metabolite, QA $[84,85]$, and xanthurenic acid (XA) [86] by KYNU and KAT I-IV, respectively. 3-Hydroxyanthranillate-3,4-dixogygenase (3-HAAO) catalyzes the conversion of 3-HANA to QA $[87,88]$. Also, a rapid reaction to an unstable intermediate product $\alpha$-amino- $\beta$-carboxymuconate- $\varepsilon$-semialdehyde (ACMS) can be followed by spontaneous rearrangement to form QA [89]. ACMS is converted to PA by ACMS decarboxylate (ACMSD). PA can be produced in human glial cells and neurons and is neuroprotective. QA is a classic example of a biochemical double-edged sword, acting as both an essential metabolite and potent neurotoxin [90-92].

Further catabolism of 3-HANA can lead to the formation of CA. CA and XA are KYN metabolites generated by oxidative dimerization of 3-HANA and transamination of 3-hydroxy-L-kynurenine (3-HK), respectively [10]. Quinolone phosphoribosyl transferase (QPRT) metabolizes QA to NAD to facilitate energy production [90-93]. However, QPRT can slow the synthesis of NAD due to its limited enzyme capacity [90].

\section{Metabolites and Molecular Mechanisms in the Kynurenine Pathway}

4.1. Kynurenine. $\mathrm{KYN}$ is the first and main metabolite of $\mathrm{L}$ TRP. The intermediate product, $\mathrm{N}$-formylkynurenine, is produced by IDO1, IDO2, and TDO and subsequently converted to KYN by formamidase [78-80]. The physiological role of KYN is not well defined. An increase in KYN levels was found incidentally in peripheral and central cells during efforts to characterize tryptophan metabolism and the associated immune response [94]. KYN scavenges hydrogen peroxide and superoxide in specific pathways that produce ROS. Increased KYN levels also lead to a decrease in ROS production by activated neutrophils [95]. KYN is used in models of neurotoxicity as a neuroprotective agent, but observed effects are attributed to its metabolite, KYNA [96-98].

IDO1 and IDO2 catalyze the rate-limiting step in the formation of KYN, especially IDO1 [78-80]. IDO1 is also a pivotal enzyme in monocytes and macrophages in the immune system and microglia and astrocytes in the nervous system. In vivo, IDO1 activity is upregulated by inflammatory factors, such as lipopolysaccharide, interferon- $\gamma$ (IFN$\gamma$ ), immune response, and chronic stress, leading to an increase in KYN levels in the peripheral and central neural tissues $[99,100]$. IFN- $\gamma$ is notably effective for inducing the 


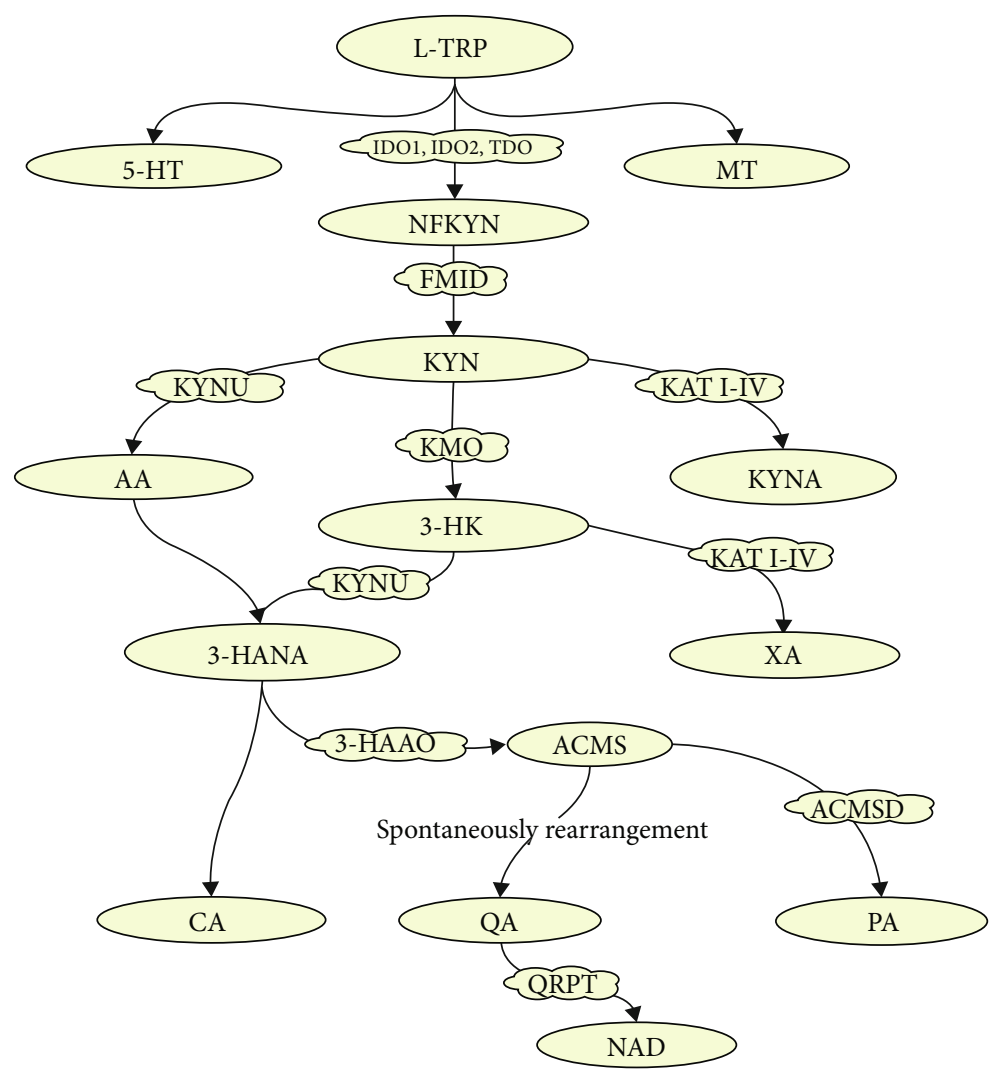

FIGURE 1: The kynurenine pathway of L-tryptophan metabolism. Green-filled ovals are the metabolic breakdown products in L-tryptophan metabolism, and cloud-like boxes filled with yellow are enzymes needed for oxidative and reductive reactions. L-TRP: L-tryptophan; 5-HT: 5-hydroxytryptophan; MT: melatonin; IDO: indoleamine-2,3-dioxygenase; TDO: tryptophan 2,3-dioxygenase; NFKYN: n-formyl-Lkynurenine; FMID: formamidase; KYN: kynurenine; KYNU: kynureninase; KMO: kynurenine 3-monooxygenase; KAT I-IV: kynurenine aminotransferase I-IV; AA: anthranilic acid; 3-HK: 3-hydroxy-L-kynurenine; KYNA: kynurenic acid; 3-HANA: 3-hydroxyanthranilic acid; XA: xanthurenic acid; 3-HAAO: 3-hydroxyanthranillate-3,4-dixogygenase; CA: cinnabarinic acid; ACMS: $\alpha$-amino- $\beta$ carboxymuconate- $\varepsilon$-semialdehyde; ACMSD: ACMS decarboxylate; QA: quinolinic acid; PA: picolinic acid; QPRT: quinolone phosphoribosyl transferase; NAD: nicotinamide adenine dinucleotide.

KP. IDO1 activity is regulated by TNF- $\alpha$, IL- $1 \alpha$, TLR, pattern-related injury, or memory recognition cells in the immune system. These factors all increase NF- $\kappa$ B signaling, which leads to further immunological dysregulation [101]. $\gamma$-[Glu]n-Trp (EW) decreases IDO activity by inhibiting inflammatory cytokine production (TNF- $\alpha$, IL- 6 , IL-1 $\beta$, and IFN- $\gamma$ ). This decrease reduces KP metabolism and restores normal behavior in mice with experimental depression [102].

4.1.1. Kynurenine/L-Tryptophan. More than $90 \%$ of L-TRP in the periphery can be converted to KYN [77]. An increase in the kynurenine/L-tryptophan $(\mathrm{K} / \mathrm{T})$ ratio may reflect an increased risk of CNS diseases and might be a useful biomarker of KP metabolic disorders [103, 104]. That is, risk increases as more L-TRP is converted to KYN. Activation of IDO1 by inflammatory cytokines can increase the production of KYN and also lead to an increased K/T ratio [99, 100]. An increased peripheral $\mathrm{K} / \mathrm{T}$ ratio implies an increase in the amount of KYN available for passive transport across the $\mathrm{BBB}[105,106]$ thus raising the concentration of KYN in the brain.
Consequently, the $\mathrm{K} / \mathrm{T}$ ratio is an important indicator of the formation of downstream metabolites in the KP. An increased $\mathrm{K} / \mathrm{T}$ ratio is a prominent feature of inflammation and mental disorders in AD studies [107-109]. Further, IDO activity can be monitored by circulating K/T [110].

4.1.2. KYN-AHR Signaling. AHR is closely associated with KYN level. AHR is a ligand-activated transcription factor and a node in the versatile intrakingdom communication system that binds molecules, such as L-TRP, with polyaromatic hydrocarbon rings $[111,112]$. AHR is a highly conserved sensor for a wide variety of chemicals from environmental pollutants to constituents of food, cells, and microorganisms. Its role is fundamental to biological processes. Dysregulation of the AHR pathway is associated with a variety of maladies, including autoimmune diseases and cancer. AHR is thus a promising target for drug development and host-directed therapy [113].

$\mathrm{KYN}$ is an endogenous ligand, exogenous receptor, and transcription factor of AHR, and a combination of the two supports the immunomodulatory role of AHR [114]. KYN activates the AHR pathway and leads to an increase in the 
TABLE 1: Summary of individual KP metabolites with known receptor targets, key biological functions, negative effects, and effects associated with $\mathrm{AD}$ (debated*).

\begin{tabular}{|c|c|c|c|c|c|}
\hline Metabolite & Receptors & Biological functions & Negative effects & Effects associated with AD & Ref. \\
\hline KYN & AHR & $\begin{array}{c}\text { Immunomodulation, anticancer, } \\
\text { oxidative stress regulation, } \\
\text { neuroprotection*, anti-inflammatory }\end{array}$ & Unknown & Antineuroinflammatory & $\begin{array}{c}{[78-80,95,} \\
99,100,119,120]\end{array}$ \\
\hline 3-HK & Unknown & Oxidative stress regulation & Neurotoxicity & Neurotoxicity & {$[96,110,125]$} \\
\hline 3-HANA & Unknown & $\begin{array}{l}\text { Anti-inflammatory, oxidative stress } \\
\text { regulation, lipid-decreasing, } \\
\text { immunomodulation }\end{array}$ & Unknown & Antineuroinflammatory & {$[127,129-135]$} \\
\hline QA & NMDAR & Proconvulsant, prooxidant & $\begin{array}{l}\text { Neurotoxicity, } \\
\text { proneuroinflammatory } \\
\text { prodepression }\end{array}$ & $\begin{array}{c}\text { Formation of } \mathrm{A} \beta \text { and } \mathrm{p} \text {-tau, } \\
\text { proneuroinflammatory } \\
\text { neurotoxicity }\end{array}$ & {$[7-9,15-23]$} \\
\hline KYNA & $\begin{array}{c}\text { AHR, } \\
\text { NMDAR, } \\
\alpha 7 \text { nAChR }\end{array}$ & $\begin{array}{l}\text { Antioxidant, immunomodulation, } \\
\text { anticonvulsant, antineurotoxicity }\end{array}$ & Unknown & Cognitive disorder & $\begin{array}{c}{[23,153-159} \\
163]\end{array}$ \\
\hline XA & $\begin{array}{l}\text { mGLUR2/ } \\
3^{*}\end{array}$ & Antioxidant & Unknown & Unknown & {$[129,165]$} \\
\hline PA & Unknown & $\begin{array}{l}\text { Anticonvulsant, antimicrobial, } \\
\text { immunomodulation }\end{array}$ & Neurotoxicity & Neurotoxicity & {$[12,167-169]$} \\
\hline CA & $\begin{array}{l}\text { AHR, } \\
\text { mGLUR4 }\end{array}$ & $\begin{array}{l}\text { Immunomodulation, } \\
\text { antineuroinflammatory }\end{array}$ & Proapoptosis & Antineuroinflammatory & {$[10,11,166]$} \\
\hline
\end{tabular}

extent of cerebral infarction in mice with experimentally induced stroke [115]. AHR inhibits the destruction and senescence of bone marrow mesenchymal stem cells induced by KYN in vitro, but underlying mechanisms are not clear [116]. The KYN-AHR signaling pathway reverses the inflammatory response of dendritic cells after exposure to lipopolysaccharide (LPS) or IFN- $\gamma[117,118]$.

Enzymes involved in the synthesis of KYN are pivotal for KYN-AHR signaling. Inhibition of IDO1 with n-acetyl 5hydroxytryptamine, a positive allosteric modulator of IDO1, can eliminate neuroinflammation in mice with experimental autoimmune encephalomyelitis (AE), a condition associated with IDO-AHR signaling [119]. In addition, inhibition of IDO1/TDO with RY103 in vitro reduced proliferation of glioma cells mediated by KYN-AHR signaling [120]. KYN-AHR signaling is important for regulating immunity, tumor, and neuroinflammation. Enzymes related to KYN play an important role in KYN/AHR signaling during neuroinflammation.

$\mathrm{KYN}$ is a critical KP metabolite for molecular metabolic changes upstream and downstream in the pathway. KYN levels are regulated by enzymes involved in responses to oxidative stress and inflammation (Table 1). Few studies are available to characterize relationships among KYN and neuroinflammation, enzymes, cytokines, and signaling pathways. Exploration of the role of KYN might be critical to understanding neuroinflammation and CNS dysfunction.

4.2. 3-Hydroxy-L-kynurenine. 3-HK is an important intermediate of the main branch of the KP pathway. It is produced by conversion of KYN by KMO [81-83]. 3-HK displays physiological characteristics similar to KYN. Thus, elevated levels of 3-HK are associated with neuroinflammation $[121,122]$. However, the role of $3-\mathrm{HK}$ in neuropathol- ogy remains poorly understood due to the relatively low number of in vivo studies that directly examine its contribution to pathology.

3-HK exhibits both antioxidation and prooxidation properties, depending on concentration. Lower concentrations are associated with strong prooxidant activity with neuronal toxicity [96] and oxidation resistance in higher concentrations [123]. 3-HK might stimulate glutathione Stransferase, superoxide dismutase, and an important transcription nuclear factor, erythroid-derived 2-like 2 (Nrf2), that is important for antioxidant regulation [121]. Therefore, altering the concentration of 3-HK may modulate antioxidant systems and activate redox cell sensing mechanisms during inflammation.

The distribution of $3-\mathrm{HK}$ is similar to that of KYN with higher concentrations in the cerebral cortex, striatum, and hippocampus [124]. Colín-González et al. injected 3-HK into a rat striatum and observed that oxidation of $3-\mathrm{HK}$ decreased with increasing time and decreasing concentration. However, a high concentration is not completely beneficial for health [121]. High doses of 3-HK administered directly to mice produced neurotoxic effects, concentration-dependent cognitive impairment, and other neuropsychiatric symptoms [110, 125]. Further, 3-HK-mediated neurotoxicity is related to toxicity dependent on QA-N-methyl-D-aspartic acid (NMDA) receptor interaction $[121,126]$. The 3-HK/QA and 3-HK/ $\mathrm{KA}$ ratios are possible biomarkers for neuropathological effects of KP metabolism [110].

4.3. 3-Hydroxyanthranilic Acid. As a precursor of QA, 3HANA is an important downstream metabolite in the KP produced by KYNU. 3-HANA regulates the release of inflammatory cytokines, counters lipid peroxidation, and lowers lipid levels in neural-immune cells [127]. 3-HANA 
levels vary among diseases for reasons that are not clear. For example, levels are lower in patients with stroke or after arterial surgery but higher in patients with Huntington's Disease (HD) or depression [128]. 3-HANA and 3-HK generate hydrogen peroxide and promote apoptosis in monocytes stimulated by IFN- $\gamma$ in vitro and in vivo. Antioxidants reduce this apoptotic effect [129-131]. 3-HANA disrupted mitochondria-mediated energy metabolism unrelated to ROS formation. 3-HK acts as an anti-inflammatory and antioxidant agent in astrocytes and microglia (Table 1). This action reflects inhibition of apoptosis in monocytes stimulated by cytokines [132] and upregulation of the antioxidant enzyme heme oxidase-1 [132]. Further, increasing the level of 3-HANA reduces the activation of macrophage inflammasomes and decreases lipid levels in obese mice [133]. 3HANA contributes to mitochondria-mediated energy metabolism unrelated to ROS formation in vitro and in vivo [134]. This factor also regulates the proliferation of $\mathrm{T}$ cells that induce inflammation in the AHR-NF- $\kappa \mathrm{B}$ signaling pathway in dendritic cells [135]. Finally, 3-HANA removes nitric oxide that can dilate blood vessels and prevent thrombosis [136].

4.4. Quinolinic acid. QA is a downstream metabolite formed from conversion of 3-HA to ACS by 3-HAAO followed by spontaneous rearrangement to QA [7]. QA is a potent neurotoxin and an essential amino acid. Microglia are responsible for QA production, and astrocytes and neurons must take up external QA to produce NAD [19, 137]. Neuroinflammation induces excess QA in activated microglia and infiltrated macrophages, which may destroy neurons, astrocytes, and oligodendrocytes [7-9]. QA exhibits neurotoxicity through at least three different mechanisms, including excitotoxicity by NMDA receptor (NMDAR) activation, formation of ROS, and destabilization of the cytoskeleton [138, 139]. Many NMDAR containing NR2A and NR2B subunits are distributed in the forebrain. QA shows a preference for binding with NMDAR, and neurons in this region are more likely to be damaged [23]. Moreover, QA can generate free radicals, increase ROS formation, and cause lipid peroxidation. These actions all increase oxidative stress [15], thereby further contributing to the pathogenesis of $\mathrm{AD}$ [140-143].

Neuropathy is accompanied by elevated levels of QA, suggesting that QA may be an important molecule in the pathogenesis of neurological diseases [144]. Structural and chemical changes in neurons after injection of QA into the brain and a QA-induced HD model have been established $[84,145,146]$. An acute QA infusion into the striatum of 30-day-old rats stimulated NFL hyperphosphorylation within $30 \mathrm{~min}$. This effect was associated with cAMPdependent protein kinase $\mathrm{A}$ and protein kinase $\mathrm{Ca} 2+/ \mathrm{cal}-$ modulin-dependent protein kinase II. Thus, both neuronal and astrocyte cytoskeletons are vulnerable to QA toxicity $[139,147]$. Intracerebroventricular injections of QA induce epileptic signs in mice and cause axon sparing lesions on neurons [23]. QA was shown to be toxic via excitation to oligodendrocytes [16] and neurons [17, 18], induced inflammation in glial cells, and promoted the occurrence of neuroinflammation [21]. QA is closely associated with the formation of $\mathrm{A} \beta$ [19] and $p$-tau [20], which are related to immune cell response in the brain. A $\beta 1-42$ directly stimulates the expression of IDO, thereby enhancing QA production in vitro and in vivo $[24,25]$. QA is also observed to induce learning and memory deficits $[20,148]$. A significant dose-dependent reduction in glutamine synthetase activity was found with QA treatment, showing that QA is an important factor for astroglial activation, dysregulation, and cell death with potential relevance to $\mathrm{AD}$ and other neuroinflammatory diseases [149]. Further, subsequent inhibition of the KP upstream of QA demonstrates that downregulating QA improves behavioral deficits in LPS hippocampal animals [125] (Table 1).

QA may also be associated with neuropsychiatric problems, possibly increasing the risk of poststroke depression [150] and increasing the risk of depressive symptoms [22]. Further, intracellular QA levels increase dramatically in response to immune stimulation (e.g., pokeweed mitogen (PWM)) in immune cells, such as dendritic cells and microglia [90], that produce less QA than macrophages [110]. Hence, QA and the KYNA/QA ratio (see Section 4.5) are both associated with immune and inflammatory responses and may be crucial therapeutic targets in degenerative diseases, such as AD.

4.5. Kynurenic acid. KYN can be converted to KYNA mediated by KAT I-IV. KYNA can only be produced by astrocytes by KAT II in the brain [98, 151, 152]. KYNA, in contrast to QA, is an antagonist at NMDA and glycine sites of this ionotropic receptor [7]. KYNA at higher concentrations modulates the production and release of glutamate. This activity is likely the primary main mechanism for KYNA-induced impairment of cognitive function [153-156]. KYNA is also an antagonist at the $\alpha 7$ nicotinic acetylcholine receptor $(\alpha 7 \mathrm{nAChR})$ and the orphan Gprotein coupled receptor 35 (GPR35) [23, 157]. Notably, KYNA might antagonize excitatory toxicity and oxidative stress caused by QA via downregulation of transcription factor, Nrf2 [158]. This metabolite also reverses cytoskeletal perturbations in striatal neurons that arise following QAinduced excitation [158]. At high concentrations, KA blocks convulsions induced by glutamate and QA [159]. Elevated levels of $\mathrm{KA}$ in the brain also lead to cognitive dysfunction in the medial prefrontal cortex (mPFC). Additionally, KAT II inhibition blocks the generation of KYNA and increases the release of glutamate in the $\mathrm{MPFC}$. This inhibition ameliorates cognitive impairment caused by excessive levels of KYNA [160-162]. KAT II-knockout mice exhibit lower levels of $\mathrm{KA}$ in the brain and improved cognition compared to control mice [162]. KYNA levels in the brain increase after immune activation of KP. Impairment of working memory follows [163], which may be related to the accumulation of KYNA in the prefrontal cortex.

4.6. Xanthurenic acid, Picolinic acid, and Cinnabarinic acid. $\mathrm{XA}$ is produced mainly in astrocytes, catalyzed by KAT II [110]. ROS clearance and Fe ion coordination complex formation with XA and KYN affect neurodegenerative diseases, but understanding this correlation is still incomplete [164]. 
XA is an antioxidant [129] and acts as an agonist at metabotropic glutamate receptors (mGLUR) mGLUR2 and mGLUR3 [165]. However, no relevant study exists that examines XA expression and AD.

CA and PA are generated by 3-HANA via two pathways. CA is oxidized by 3-HANA to produce superoxide anion and hydrogen peroxide, which induces ROS formation and caspase activation, inhibits the mitochondrial respiratory chain, and promotes apoptosis [11]. CA is an agonist of mGLUR4 [10]. CA levels in the brain are typically low, which seems to protect neurons. These levels may increase after immune stimulation [10]. This response might modulate immune responses, such as binding to immune receptors, such as AHR. This binding may regulate $\mathrm{T}$ cells and reduce neuroinflammation [166] with therapeutic implications for $\mathrm{AD}$. PA is an unstable intermediate of 3-HANA decomposition produced by ACMSD. The production of PA is positively correlated with ACMSD activity [22, 89]. Generally, the concentration of PA in the periphery is higher than that in the CNS; PA levels can peak in adulthood but decline over time [93]. PA may have a dual function of immune regulation that induces or suppresses the expression of immune cells, such as macrophage proteins MIP$1 \alpha$ and MIP- $1 \beta$, and T cells $[12,167,168]$. High doses of PA are neurotoxic but can reduce the excitatory toxicity of QA [12]. Further, PA has an antimicrobial function [169] (Table 1).

\section{KP Metabolites as Biomarkers in AD}

Current diagnostic methods for dementia are notoriously complex, involving expensive PET or MRI scans or CSF tests. Resources for these diagnostics, for health professionals trained to provide them, are limited globally [1]. Metabolomics is an approach that complements hypothesis-driven techniques and targets. Specific factors, with known associations, such as molecular signals (e.g., APOE $\varepsilon 4$ ), are controversial targets for $\mathrm{AD}$ patients $[170,171]$. Many pathways-lipid, uric acid, and amino acid metabolism [172-176], immune and inflammatory responses [177], BBB dysfunction, and metal ion homeostasis-can modulate $\mathrm{AD}$. $\mathrm{AD}$, as a multifactorial neurodegenerative disease associated with aging, requires careful diagnosis to accurately distinguish it from normal aging. Therefore, finding biomarkers related to $\mathrm{AD}$ is critical for the prevention, diagnosis, and treatment of $\mathrm{AD}$.

Serum, CSF, and imaging examinations are often used for the diagnosis of $\mathrm{AD}$ patients. However, in most animal studies, pathological sections of brain tissue and CSF are generally used to detect known pathological products and possible biomarkers. In 2018, biomarkers were grouped into A (amyloid), T ( $p$-tau), and N (neurodegeneration, measured by total tau(t-tau) where applicable) [178]. Differences between KP peripheral and central metabolism reflect varied metabolite function in the pathway. Consequently, both serum and CSF were analyzed to find biomarkers to aid $\mathrm{AD}$ diagnosis.

A large-scale multicenter study in Europe assessed metabolic phenotypes of urine and serum from the Europewide AddNeuroMed/Dementia Case Register biobank of patients clinically diagnosed with $\mathrm{AD}$ and MCI. 5-HT (urine, serum), KYN (serum), KYNA (urine), L-TRP (urine, serum), and $\mathrm{K} / \mathrm{T}$ ratio (urine) were significantly lower in $\mathrm{AD}$ patients than in controls. The bioavailability of neurotransmitters in $\mathrm{AD}$ may be altered. Further, the significant increase in the $\mathrm{K} / \mathrm{T}$ ratio might be due to increased activity of IDO and more active metabolism of KP associated with systemic inflammation [179].

The levels of KYNA in the CSF of AD patients are significantly increased compared with normal controls [13, 107]. Increased KYNA concentrations in CSF in patients with $\mathrm{AD}$ are not seen in other neurodegenerative diseases, such as frontotemporal dementia (FTD), amyotrophic lateral sclerosis, and progressive supranuclear palsy. KYNA may be specifically elevated in AD [180].

KYN and 5-HT are metabolites of L-TRP, and KYN is the main product produced by activation of KP. Patients with normal cognition, MCI, and AD were assessed in a clinical study for KYN, L-TRP, and 5-HT in serum, A $\beta$, and tau and $\mathrm{KYN}$ in CSF. An inflammatory signal cascade may occur during $\mathrm{AD}$, related to the increase in KYN [181]. Higher K/T ratios were associated with many inflammatory markers and lower functional independence and memory scores. A $\beta$ and complement systems may be key contributing factors in this process [181].

Plasma concentrations of KYN, 3-HANA, XA, QA, and $\mathrm{L}$-TRP were found to be lower in $\mathrm{AD}$ patients compared to controls [109]. Specially, elevated QA was associated with poor cognition in older AD patients [109]. Another study found that the plasma concentrations of L-TRP and KYNA decreased and $\mathrm{QA}$ increased in $\mathrm{AD}$, which proved the activation of peripheral KP in this type of dementia [182]. Plasma $\mathrm{KYN}$ and $\mathrm{PA}$ in $\mathrm{AD}$ patients were inversely correlated with CSF $p$-tau and $t$-tau, respectively. Moreover, increased 3$\mathrm{HK} / \mathrm{KYN}$ ratios correlated with $t$-tau in CSF of AD patients [107]. Further, L-TRP catabolites, KYNA and QA, showed significantly higher concentrations in the CSF of AD patients. These higher concentrations were correlated, as were other L-TRP pathway intermediates, with either CSF A $\beta 1-42$ or tau and p-tau-181 [13]. Concentrations of KYNA were reduced in the CSF of AD patients. Also, serum KYN and QA levels strongly correlated with their respective content in CSF, and KYN in serum was negatively correlated with $\mathrm{AD}$ disease severity. Also, it has been suggested that the development of inflammatory-mediated neuropathology is correlated with changes in the ratio of KYNA to QA rather than QA levels alone [183]. KYN metabolites accumulated similarly with aging in serum and CSF in AD patients and control subjects. In contrast, KYNA was significantly reduced in the CSF of $\mathrm{AD}$ patients. Age- and diseasespecific changes in cerebral $\mathrm{KP}$ activity might contribute to reduced neurogenesis and increased excitotoxicity in neurodegenerative disease [184].

These findings support KP involvement in $\mathrm{AD}$ pathogenesis and support the concept of therapeutic modulation of KP for the treatment of AD. Further, KYN (plasma, serum, and CSF), KYNA (serum, urine, and CSF), L-TRP (plasma, serum, and urine), K/T ratio (plasma, serum, urine, and CSF), 3-HANA (plasma), XA (plasma), and QA 
TABLE 2: Studies of biomarkers in biofluids and their association with KP metabolites in AD.

\begin{tabular}{|c|c|c|c|}
\hline $\begin{array}{l}\text { Biofluid } \\
\text { biomarker }\end{array}$ & Metabolites & Observation & Ref. \\
\hline \multirow[b]{2}{*}{ Serum } & 17 metabolites & Decreased concentration of L-TRP, KYN, and KYNA & {$[179]$} \\
\hline & $\begin{array}{c}\text { L-TRP, KYN, and 5- } \\
\text { HT }\end{array}$ & Increased concentration of $\mathrm{K} / \mathrm{T}$ & {$[181]$} \\
\hline \multirow[b]{2}{*}{ Plasma } & 17 metabolites & Decreased concentration of KYN, 3-HANA, XA, QA, and L-TRP & [109] \\
\hline & 6 metabolites & $\begin{array}{l}\text { Decreased concentration of L-TRP and KYNA; increased concentration of } \\
\text { QA }\end{array}$ & {$[182]$} \\
\hline Urine & 8 metabolites & Decreased concentration of KYNA, L-TRP, and K/T & [179] \\
\hline \multirow{2}{*}{ CSF } & L-TRP & Increased concentration of KYNA & {$[13,107,180]$} \\
\hline & L-TRP & Increased concentration of KYNA and QA & [13] \\
\hline CSF and serum & $\begin{array}{l}\text { L-TRP, KYN, and 5- } \\
\text { HT }\end{array}$ & Increased concentration of $\mathrm{KYN}$ & {$[181]$} \\
\hline
\end{tabular}

(plasma, CSF) were found to be related to the severity of AD. Metabolites of KP, such as KYN and QA, may be useful biomarkers for the early diagnosis of AD. Table 2 lists biomarker studies in biological fluids that suggest notable associations of KP metabolites with $\mathrm{AD}$.

\section{Therapeutic Considerations}

NMDAR blockers, such as MK-801 and memantine, reduce the glutamate neurotoxicity [185]. Memantine, commonly used for the treatment of $\mathrm{AD}$, acts as an NMDAR antagonist and reduces QA-induced hippocampal injury [186]. KP metabolism has an important influence on the occurrence of $\mathrm{AD}$ and may be an important target for the treatment of AD. Changes in KYNA and QA are associated with cognitive impairment in $\mathrm{AD}$ patients [182]. QA is neurotoxic, while KYNA may exhibit neuroprotective effects [187]. The systemic administration of 4-chlorokynurenine (4-Cl-KYN), an NMDAR, did prevent QA-induced neurotoxicity in the hippocampus of the rats [188]. 4-Cl-KYN can be transformed into 7-chlorokynurenic acid (4-Cl-3-HANA), and thus, it can inhibit 3-HANA oxygenase and QA synthesis [189]. Therefore, modification of the KP through pharmacological inhibition of the enzymes of QA synthesis is a rational approach via which to divert the KP metabolism toward the neuroprotective KYNA [190]. Also, upregulating KYNA synthesis and reducing KP metabolites formed by QA may be a useful therapeutic strategy.

Regulating KP activity may improve some symptoms of $\mathrm{AD}$ in animal models [3, 191-193]. Inhibition of KYN metabolism in the blood to increase KYN transport to the brain increases brain KYNA production. This action prevents synaptic loss and improves memory function in mice with experimental AD [192].

In addition, intervention at the level of KP-related enzymes may also be important for $\mathrm{AD}$ treatment. $\mathrm{A} \beta 1-42$ directly stimulates IDO expression, thereby enhancing QA production in vitro and in vivo $[24,25]$. The IDO inhibitor, coptisine, ameliorated AD-like signs in a mouse model of AD. Coptisine inhibition of IDO decreases the activation of microglia and astrocytes, consequently improving neuronal loss and $\mathrm{A} \beta$ formation [194]. Cognition, anxiety, and depression improved in 3XTG-AD mice treated with the novel IDO inhibitor, DWG-1036 [195]. Similarly, TDO inhibition reduces neurodegeneration in animal models of $\mathrm{AD}$ [196]. TDO is highly expressed in the brain tissues of both $\mathrm{AD}$ model mice and $\mathrm{AD}$ patients, suggesting that TDO-mediated KP activation may be involved in the formation of neurofibrillary tangles and senile plaques [197]. In addition, treatment of APP/PS1 mice with a TDO inhibitor for 4 weeks prevented deficits in hippocampal-dependent memory function [198]. Finally, inhibition of KMO reduces synaptic loss in a mouse AD model. Treatment of AD mice significantly improved symptoms and delayed disease progression [192, 199]. Thus, KP metabolites and enzymes are clinically significant targets for the treatment of AD.

\section{Concluding Remarks}

L-TRP metabolism by the KP regulates inflammation, immunity, and tumor progression. KP signaling is the result of various metabolic influences, such as inflammatory factors, and the early discovery of metabolites can support the early diagnosis and treatment of AD. Firstly, studies suggested that peripheral inflammatory factors played a key role in the early stage of the blood-cerebrospinal fluid barrier [5, 26]. Therefore, we should take a holistic view of inflammation that peripheral inflammation was associated with neuroinflammation. Secondly, KP metabolites and molecular mechanisms are complex and most of them have yet to be explored. Different biomarkers are the most appropriate from the perspective of molecular mechanisms and existing biomarker exploration. KYN, 3-HANA, and CA have an antineuroinflammatory effect which can inhibit $\mathrm{AD}$. It is found that KYNA can lead to cognitive impairment. 3-HK, $\mathrm{QA}$, and PA have neurotoxicity and promote AD. QA is also supposed to be closely related to the formation of $A \beta$ and $p$ tau and to promote neuroinflammation. According to existing studies, elevated QA promotes pathological changes and neuroinflammation in AD. However, KYN might be more 
advantageous as a biomarker in related studies. This may be because QA poorly diffuses across the BBB and mainly exists in the CNS, while KYN can cross the BBB through the large neutral amino acid transporter. We consider that KYN might be more advantageous as a biomarker, while QA might be more advantageous as a therapeutic target. Therefore, future studies should carefully explore the biological functions of various important KP metabolites. Further mechanistic studies are needed to link KP metabolites with $\mathrm{AD}$. However, modulation of the $\mathrm{KP}$ at various points in the pathway is likely to be beneficial. KP metabolites, enzyme inhibitors, and neutralizing antibodies are future therapeutic strategies in neurological disease.

\section{Conflicts of Interest}

The authors declare no competing financial interests.

\section{Authors' Contributions}

All the authors contributed equally to this work.

\section{Acknowledgments}

This work was supported by grants from the National Key R\&D Program of China (Nos. 2020YFC2003100 and 2020YFC2003104), the "Xing-lin Scholars" Project of Chengdu University of Traditional Chinese Medicine (No. QNXZ2018004), and the Third Batch of Major Science and Technology Application Demonstration Projects in Chengdu (No. 2019-YF09-00096-SN).

\section{References}

[1] S. Gauthier, P. Rosa-Neto, J. A. Morais, and C. Webster, World Alzheimer Report 2021: Journey through the Diagnosis of Dementia, Alzheimer's Disease International, London, England, 2021.

[2] S. van der Lee, F. Wolters, M. Ikram et al., "The effect of $A P O E$ and other common genetic variants on the onset of Alzheimer's disease and dementia: a community-based cohort study," The Lancet Neurology, vol. 17, no. 5, pp. 434-444, 2018.

[3] J. Yu, L. Tan, and J. Hardy, "Apolipoprotein E in Alzheimer's disease: an update," Annual Review of Neuroscience, vol. 37, no. 1, pp. 79-100, 2014.

[4] K. Lai, C. Liu, A. Rau et al., "Peripheral inflammatory markers in Alzheimer's disease: a systematic review and meta-analysis of 175 studies," Journal of Neurology, Neurosurgery, and Psychiatry, vol. 88, no. 10, pp. 876-882, 2017.

[5] X. Shen, L. Niu, Y. Wang et al., "Inflammatory markers in Alzheimer's disease and mild cognitive impairment: a metaanalysis and systematic review of 170 studies," Journal of Neurology, Neurosurgery, and Psychiatry, vol. 90, no. 5, pp. 590-598, 2019.

[6] C. Wolfe, N. Fitz, K. Nam, I. Lefterov, and R. Koldamova, "The role of Apoe and TREM2 in Alzheimer's diseasecurrent understanding and perspectives," International Journal of Molecular Sciences, vol. 20, no. 1, p. 81, 2019.

[7] G. Guillemin, "Quinolinic acid, the inescapable neurotoxin," The FEBS Journal, vol. 279, no. 8, pp. 1356-1365, 2012.
[8] T. Stone, "Endogenous neurotoxins from tryptophan," Toxicon, vol. 39, no. 1, pp. 61-73, 2001.

[9] R. Lugo-Huitrón, P. Ugalde Muñiz, B. Pineda, J. PedrazaChaverrí, C. Ríos, and V. Pérez-de la Cruz, "Quinolinic acid: an endogenous neurotoxin with multiple targets," Oxidative Medicine and Cellular Longevity, vol. 2013, Article ID 104024, 14 pages, 2013.

[10] F. Fazio, L. Lionetto, M. Curto et al., "Cinnabarinic acid and xanthurenic acid: two kynurenine metabolites that interact with metabotropic glutamate receptors," Neuropharmacology, vol. 112, pp. 365-372, 2017.

[11] Y. Nagamura, K. Uesugi, J. Naito, and I. Ishiguro, "Cinnabarinic acid was formed in damaged mitochondria and its effect on mitochondrial respiration," Advances in Experimental Medicine and Biology, vol. 467, pp. 419-423, 1999.

[12] L. Vrooman, K. Jhamandas, R. Boegman, and R. Beninger, "Picolinic acid modulates kainic acid-evoked glutamate release from the striatum in vitro," Brain Research, vol. 627, no. 2, pp. 193-198, 1993.

[13] V. van der Velpen, T. Teav, H. Gallart-Ayala et al., "Systemic and central nervous system metabolic alterations in Alzheimer's disease," Alzheimer's Research \& Therapy, vol. 11, no. 1, p. 93, 2019.

[14] Y. Muguruma, H. Tsutsui, T. Noda, H. Akatsu, and K. Inoue, "Widely targeted metabolomics of Alzheimer's disease postmortem cerebrospinal fluid based on 9-fluorenylmethyl chloroformate derivatized ultra-high performance liquid chromatography tandem mass spectrometry," Journal of Chromatography, vol. 1091, pp. 53-66, 2018.

[15] A. Santamaría, S. Galván-Arzate, V. Lisý et al., "Quinolinic acid induces oxidative stress in rat brain synaptosomes," $\mathrm{Neu}$ roreport, vol. 12, no. 4, pp. 871-874, 2001.

[16] G. Sundaram, B. Brew, S. Jones, S. Adams, C. Lim, and G. Guillemin, "Quinolinic acid toxicity on oligodendroglial cells: relevance for multiple sclerosis and therapeutic strategies," Journal of Neuroinflammation, vol. 11, no. 1, p. 204, 2014.

[17] S. Kerr, P. Armati, G. Guillemin, and B. Brew, "Chronic exposure of human neurons to quinolinic acid results in neuronal changes consistent with AIDS dementia complex," AIDS (London, England), vol. 12, no. 4, pp. 355-363, 1998.

[18] Y. Chen, B. Brew, and G. Guillemin, "Characterization of the kynurenine pathway in Nsc-34 cell line: implications for amyotrophic lateral sclerosis," Journal of Neurochemistry, vol. 118, no. 5, pp. 816-825, 2011.

[19] G. Guillemin, B. Brew, C. Noonan, O. Takikawa, and K. Cullen, "Indoleamine 2,3 dioxygenase and quinolinic acid immunoreactivity in Alzheimer's disease hippocampus," Neuropathology and Applied Neurobiology, vol. 31, no. 4, pp. 395-404, 2005.

[20] A. Rahman, M. Rao, and K. Khan, "Intraventricular infusion of quinolinic acid impairs spatial learning and memory in young rats: a novel mechanism of lead-induced neurotoxicity," Journal of Neuroinflammation, vol. 15, no. 1, p. 263, 2018.

[21] G. Guillemin, J. Croitoru-Lamoury, D. Dormont, P. Armati, and B. Brew, "Quinolinic acid upregulates chemokine production and chemokine receptor expression in astrocytes," Glia, vol. 41, no. 4, pp. 371-381, 2003.

[22] L. Brundin, C. Sellgren, C. Lim et al., "An enzyme in the kynurenine pathway that governs vulnerability to suicidal 
behavior by regulating excitotoxicity and neuroinflammation," Translational Psychiatry, vol. 6, no. 8, article e865, 2016.

[23] T. Stone and M. Perkins, "Quinolinic acid: a potent endogenous excitant at amino acid receptors in CNS," European Journal of Pharmacology, vol. 72, no. 4, pp. 411-412, 1981.

[24] G. Guillemin, G. Smythe, L. Veas, O. Takikawa, and B. Brew, "A $\beta 1-42$ induces production of quinolinic acid by human macrophages and microglia," Neuroreport, vol. 14, no. 18, pp. 2311-2315, 2003.

[25] L. Souza, C. Jesse, M. Antunes et al., "Indoleamine-2,3-dioxygenase mediates neurobehavioral alterations induced by an intracerebroventricular injection of amyloid- $\beta 1-42$ peptide in mice," Brain, Behavior, and Immunity, vol. 56, pp. 363377, 2016.

[26] D. DiSabato, N. Quan, and J. Godbout, "Neuroinflammation: the devil is in the details," Journal of Neurochemistry, vol. 139, pp. 136-153, 2016.

[27] D. Norden, P. Trojanowski, E. Villanueva, E. Navarro, and J. Godbout, "Sequential activation of microglia and astrocyte cytokine expression precedes increased Iba-1 or Gfap immunoreactivity following systemic immune challenge," Glia, vol. 64, no. 2, pp. 300-316, 2016.

[28] A. Dunn, A. Swiergiel, H. Zhang, and N. Quan, "Reduced ingestion of sweetened milk induced by interleukin-1 and lipopolysaccharide is associated with induction of cyclooxygenase-2 in brain endothelia," Neuroimmunomodulation, vol. 13, no. 2, pp. 96-104, 2006.

[29] F. Leng and P. Edison, "Neuroinflammation and microglial activation in Alzheimer disease: where do we go from here?," Nature Reviews Neurology, vol. 17, no. 3, pp. 157-172, 2021.

[30] T. Owens, R. Khorooshi, A. Wlodarczyk, and N. Asgari, "Interferons in the central nervous system: a few instruments play many tunes," Glia, vol. 62, no. 3, pp. 339-355, 2014.

[31] S. Solé-Domènech, D. Cruz, E. Capetillo-Zarate, and F. Maxfield, "The endocytic pathway in microglia during health, aging and Alzheimer's disease," Ageing Research Reviews, vol. 32, pp. 89-103, 2016.

[32] P. McGeer, S. Itagaki, H. Tago, and E. McGeer, "Reactive microglia in patients with senile dementia of the Alzheimer type are positive for the histocompatibility glycoprotein HlaDr," Neuroscience Letters, vol. 79, no. 1-2, pp. 195-200, 1987.

[33] E. Spangenberg and K. Green, "Inflammation in Alzheimer's disease: lessons learned from microglia-depletion models," Brain, Behavior, and Immunity, vol. 61, pp. 1-11, 2017.

[34] E. Martin, C. Boucher, B. Fontaine, and C. Delarasse, "Distinct inflammatory phenotypes of microglia and monocytederived macrophages in Alzheimer's disease models: effects of aging and amyloid pathology," Aging Cell, vol. 16, no. 1, pp. 27-38, 2017.

[35] H. Akiyama, S. Barger, S. Barnum et al., "Inflammation and Alzheimer's disease," Neurobiology of Aging, vol. 21, no. 3, pp. 383-421, 2000.

[36] R. del Bo, N. Angeretti, E. Lucca, M. G. de Simoni, and G. Forloni, "Reciprocal control of inflammatory cytokines, Il- 1 and Il-6, and $\beta$-amyloid production in cultures," Neuroscience Letters, vol. 188, no. 1, pp. 70-74, 1995.

[37] S. Alawieyah Syed Mortadza, J. Sim, V. Neubrand, and L. Jiang, "A critical role of Trpm 2 channel in A $\beta 42$-induced microglial activation and generation of tumor necrosis factor- $A$," Glia, vol. 66, no. 3, pp. 562-575, 2018.
[38] C. Venegas and M. Heneka, "Danger-associated molecular patterns in Alzheimer's disease," Journal of Leukocyte Biology, vol. 101, no. 1, pp. 87-98, 2017.

[39] S. Liu, Y. Liu, W. Hao et al., "Tlr2 is a primary receptor for Alzheimer's amyloid $\beta$ peptide to trigger neuroinflammatory activation," Journal of Immunology (Baltimore, Md. : 1950), vol. 188, no. 3, pp. 1098-1107, 2012.

[40] H. Keren-Shaul, A. Spinrad, A. Weiner et al., "A unique microglia type associated with restricting development of Alzheimer's disease," Cell, vol. 169, no. 7, pp. 1276-90.e17, 2017.

[41] T. Jay, V. von Saucken, and G. Landreth, "TREM2 in neurodegenerative diseases," Molecular Neurodegeneration, vol. 12, no. 1, p. 56, 2017.

[42] F. Yeh, D. Hansen, and M. Sheng, "TREM2, microglia, and neurodegenerative diseases," Trends in Molecular Medicine, vol. 23, no. 6, pp. 512-533, 2017.

[43] M. Sekiya, M. Wang, N. Fujisaki et al., "Integrated biology approach reveals molecular and pathological interactions among Alzheimer's A $\beta 42$, tau, TREM2, and TYROBP in Drosophila models," Genome Medicine, vol. 10, no. 1, p. 26, 2018.

[44] For the Alzheimer's Disease Neuroimaging Initiative, H. Takahashi, Z. A. Klein et al., "Opposing effects of progranulin deficiency on amyloid and tau pathologies via microglial TYROBP network," Acta Neuropathologica, vol. 133, no. 5, pp. 785-807, 2017.

[45] D. Norden and J. Godbout, "Review: microglia of the aged brain: primed to be activated and resistant to regulation," Neuropathology and Applied Neurobiology, vol. 39, no. 1, pp. 19-34, 2013.

[46] B. Spittau, "Aging microglia-phenotypes, functions and implications for age-related neurodegenerative diseases," Frontiers in Aging Neuroscience, vol. 9, p. 194, 2017.

[47] L. Hamelin, J. Lagarde, G. Dorothée et al., "Early and protective microglial activation in Alzheimer's disease: a prospective study using18F-DPA-714 PET imaging," Brain: a Journal of Neurology, vol. 139, no. 4, pp. 1252-1264, 2016.

[48] M. Liu, S. Jevtic, K. Markham-Coultes et al., "Investigating the Efficacy of a Combination A $\beta$-Targeted Treatment in a Mouse Model of Alzheimer's Disease," Brain Research, vol. 1678, pp. 138-145, 2018.

[49] R. Bernhardi and J. Nicholls, "Transformation of leech microglial cell morphology and properties following coculture with injured central nervous system tissue," The Journal of Experimental Biology, vol. 202, no. 6, pp. 723-728, 1999.

[50] K. Townsend, T. Town, T. Mori et al., "Cd40 signaling regulates innate and adaptive activation of microglia in response to amyloid ?-peptide," European Journal of Immunology, vol. 35, no. 3, pp. 901-910, 2005.

[51] E. Dursun, D. Gezen-Ak, H. Hanağası et al., "The interleukin 1 alpha, interleukin 1 beta, interleukin 6 and alpha-2macroglobulin serum levels in patients with early or late onset Alzheimer's disease, mild cognitive impairment or Parkinson's disease," Journal of Neuroimmunology, vol. 283, pp. 50-57, 2015.

[52] C. Jack, D. Knopman, W. Jagust et al., "Tracking pathophysiological processes in Alzheimer's disease: an updated hypothetical model of dynamic biomarkers," The Lancet Neurology, vol. 12, no. 2, pp. 207-216, 2013. 
[53] K. Westin, P. Buchhave, H. Nielsen, L. Minthon, S. Janciauskiene, and O. Hansson, "Ccl2 is associated with a faster rate of cognitive decline during early stages of Alzheimer's disease," PloS One, vol. 7, no. 1, article e30525, 2012.

[54] L. Harries, R. Bradley-Smith, D. Llewellyn et al., "LeukocyteCCR2Expression is associated with Mini-Mental State Examination score in older adults," Rejuvenation Research, vol. 15, no. 4, pp. 395-404, 2012.

[55] E. Zenaro, E. Pietronigro, V. D. Bianca et al., "Neutrophils promote Alzheimer's disease-like pathology and cognitive decline via LFA-1 integrin," Nature Medicine, vol. 21, no. 8, pp. 880-886, 2015.

[56] C. Mai, Z. Tan, Y. Xu et al., "CXCL12-mediated monocyte transmigration into brain perivascular space leads to neuroinflammation and memory deficit in neuropathic pain," Theranostics, vol. 11, no. 3, pp. 1059-1078, 2021.

[57] P. Brzdak, D. Nowak, G. Wiera, and J. Mozrzymas, "Multifaceted roles of metzincins in CNS physiology and pathology: from synaptic plasticity and cognition to neurodegenerative disorders," Frontiers in Cellular Neuroscience, vol. 11, p. 178, 2017.

[58] E. Candelario-Jalil, Y. Yang, and G. Rosenberg, "Diverse roles of matrix metalloproteinases and tissue inhibitors of metalloproteinases in neuroinflammation and cerebral ischemia," Neuroscience, vol. 158, no. 3, pp. 983-994, 2009.

[59] T. Behl, G. Kaur, A. Sehgal et al., "Multifaceted role of matrix metalloproteinases in neurodegenerative diseases: pathophysiological and therapeutic perspectives," International Journal of Molecular Sciences, vol. 22, no. 3, p. 1413, 2021.

[60] Y. Chen, X. He, S. Wang, and Y. Xia, " $\delta$-Opioid receptors, microRNAs, and neuroinflammation in cerebral ischemia/ hypoxia," Frontiers in Immunology, vol. 11, p. 421, 2020.

[61] S. Sarkar, A. Russell, E. Engler-Chiurazzi, K. Porter, and J. Simpkins, "MicroRNAs and the genetic nexus of brain aging, neuroinflammation, neurodegeneration, and brain trauma," Aging and Disease, vol. 10, no. 2, pp. 329-352, 2019.

[62] J. Cheng, Y. Dong, J. Ma et al., "Microglial Calhm2 regulates neuroinflammation and contributes to Alzheimer's disease pathology," Science Advances, vol. 7, no. 35, 2021.

[63] I. Chen and T. Ichinohe, "Response of host inflammasomes to viral infection," Trends in Microbiology, vol. 23, no. 1, pp. 55-63, 2015.

[64] A. Lopez-Rodriguez, E. Hennessy, C. Murray et al., “Acute systemic inflammation exacerbates neuroinflammation in Alzheimer's disease: Il-1 $\beta$ drives amplified responses in primed astrocytes and neuronal network dysfunction," Alzheimer's \& Dementia, vol. 17, no. 10, pp. 1735-1755, 2021.

[65] E. Spangenberg, P. Severson, L. Hohsfield et al., "Sustained microglial depletion with CSF1R inhibitor impairs parenchymal plaque development in an Alzheimer's disease model," Nature Communications, vol. 10, no. 1, p. 3758, 2019.

[66] L. Friker, H. Scheiblich, I. Hochheiser et al., " $\beta$-Amyloid clustering around ASC fibrils boosts its toxicity in microglia," Cell Reports, vol. 30, no. 11, pp. 3743-3754.e6, 2020.

[67] C. Ising, C. Venegas, S. Zhang et al., "NLRP3 inflammasome activation drives tau pathology," Nature, vol. 575, no. 7784, pp. 669-673, 2019.

[68] W. Lin, M. Ding, J. Xue, and W. Leng, "The role of TLR2/ JNK/NF- $\kappa$ B pathway in amyloid $\beta$ peptide-induced inflammatory response in mouse Ng108-15 neural cells," International Immunopharmacology, vol. 17, no. 3, pp. 880-884, 2013.
[69] N. Lonnemann, S. Hosseini, C. Marchetti et al., "The NLRP3 inflammasome inhibitor OLT1177 rescues cognitive impairment in a mouse model of Alzheimer's disease," Proceedings of the National Academy of Sciences of the United States of America, vol. 117, no. 50, pp. 32145-32154, 2020.

[70] E. Katsyuba, M. Romani, D. Hofer, and J. Auwerx, "NAD homeostasis in health and disease," Nature Metabolism, vol. 2, no. 1, pp. 9-31, 2020.

[71] H. Grifka-Walk, B. Jenkins, and D. Kominsky, "Amino acid Trp: the far out impacts of host and commensal tryptophan metabolism," Frontiers in Immunology, vol. 12, article 653208, 2021.

[72] L. Zhang and S. Davies, "Microbial metabolism of dietary components to bioactive metabolites: opportunities for new therapeutic interventions," Genome Medicine, vol. 8, no. 1, p. 46, 2016.

[73] J. Lee, T. Wood, and J. Lee, "Roles of indole as an interspecies and interkingdom signaling molecule," Trends in Microbiology, vol. 23, no. 11, pp. 707-718, 2015.

[74] S. Jones, G. Guillemin, and B. Brew, "The kynurenine pathway in stem cell biology," International Journal of Tryptophan Research: IJTR, vol. 6, article IJTR.S12626, 2013.

[75] L. Hu, X. Li, J. Hu et al., "A simple HPLC-MS/MS method for determination of tryptophan, kynurenine and kynurenic acid in human serum and its potential for monitoring antidepressant therapy," Journal of Analytical Toxicology, vol. 41, no. 1, pp. 37-44, 2017.

[76] M. Sakurai, Y. Yamamoto, N. Kanayama et al., "Serum metabolic profiles of the tryptophan-kynurenine pathway in the high risk subjects of major depressive disorder," Scientific Reports, vol. 10, no. 1, p. 1961, 2020.

[77] T. Stone, C. Forrest, and L. Darlington, "Kynurenine pathway inhibition as a therapeutic strategy for neuroprotection," The FEBS Journal, vol. 279, no. 8, pp. 1386-1397, 2012.

[78] H. Ball, H. Yuasa, C. Austin, S. Weiser, and N. Hunt, "Indoleamine 2,3-dioxygenase-2; a new enzyme in the kynurenine pathway," The International Journal of Biochemistry \& Cell Biology, vol. 41, no. 3, pp. 467-471, 2009.

[79] N. King and S. Thomas, "Molecules in focus: indoleamine 2,3-dioxygenase," The International Journal of Biochemistry \& Cell Biology, vol. 39, no. 12, pp. 2167-2172, 2007.

[80] L. Capece, A. Lewis-Ballester, M. Marti, D. Estrin, and S. Yeh, "Molecular basis for the substrate stereoselectivity in tryptophan dioxygenase," Biochemistry, vol. 50, no. 50, pp. 1091010918, 2011.

[81] R. Brouns, R. Verkerk, T. Aerts et al., “The role of tryptophan catabolism along the kynurenine pathway in acute ischemic stroke," Neurochemical Research, vol. 35, no. 9, pp. 1315$1322,2010$.

[82] L. Amori, P. Guidetti, R. Pellicciari, Y. Kajii, and R. Schwarcz, "On the relationship between the two branches of the kynurenine pathway in the rat brainin vivo," Journal of Neurochemistry, vol. 109, no. 2, pp. 316-325, 2009.

[83] K. Jacobs, G. Castellano-Gonzalez, G. Guillemin, and D. Lovejoy, "Major developments in the design of inhibitors along the kynurenine pathway," Current Medicinal Chemistry, vol. 24, pp. 2471-2495, 2017.

[84] R. Schwarcz, W. Whetsell, and R. Mangano, "Quinolinic acid: an endogenous metabolite that produces axon-sparing lesions in rat brain," Science (New York, N.Y.), vol. 219, no. 4582 , pp. $316-318,1983$. 
[85] G. Guillemin, D. Smith, G. Smythe, P. Armati, and B. Brew, "Expression of the kynurenine pathway enzymes in human microglia and macrophages," Advances in Experimental Medicine and Biology, vol. 527, pp. 105-112, 2003.

[86] O. Taleb, M. Maammar, C. Klein, M. Maitre, and A. MensahNyagan, "A role for xanthurenic acid in the control of brain dopaminergic activity," International Journal of Molecular Sciences, vol. 22, no. 13, p. 6974, 2021.

[87] A. Foster, R. White, and R. Schwarcz, "Synthesis of quinolinic acid by 3-hydroxyanthranilic acid oxygenase in rat brain tissue in vitro," Journal of Neurochemistry, vol. 47, no. 1, pp. 2330, 1986.

[88] X. Wang, I. Davis, A. Liu, and S. Shamsi, "Development of a CZE-ESI-MS assay with a sulfonated capillary for profiling picolinic acid and quinolinic acid formation in multienzyme system," Electrophoresis, vol. 34, no. 12, pp. 1828-1835, 2013.

[89] L. Pucci, S. Perozzi, F. Cimadamore, G. Orsomando, and N. Raffaelli, "Tissue expression and biochemical characterization of human 2-amino 3-carboxymuconate 6-semialdehyde decarboxylase, a key enzyme in tryptophan catabolism," The FEBS Journal, vol. 274, no. 3, pp. 827-840, 2007.

[90] J. Moffett, P. Arun, N. Puthillathu et al., "Quinolinate as a marker for kynurenine metabolite formation and the unresolved question of $\mathrm{NAD}^{+}$synthesis during inflammation and infection," Frontiers in Immunology, vol. 11, p. 31, 2020.

[91] Y. Nishizuka and O. Hayaishi, "Studies on the biosynthesis of nicotinamide adenine dinucleotide: I. Enzymic synthesis of niacin ribonucleotides from 3-hydroxyanthranilic acid in mammalian tissues," The Journal of Biological Chemistry, vol. 238, no. 10, pp. 3369-3377, 1963.

[92] D. Pawlak, K. Pawlak, J. Malyszko, M. Mysliwiec, and W. Buczko, "Accumulation of toxic products degradation of kynurenine in hemodialyzed patients," International Urology and Nephrology, vol. 33, no. 2, pp. 399-404, 2001.

[93] N. Braidy, G. Guillemin, H. Mansour, T. Chan-Ling, and R. Grant, "Changes in kynurenine pathway metabolism in the brain, liver and kidney of aged female Wistar rats," The FEBS Journal, vol. 278, no. 22, pp. 4425-4434, 2011.

[94] C. Opitz, U. Litzenburger, F. Sahm et al., "An endogenous tumour-promoting ligand of the human aryl hydrocarbon receptor," Nature, vol. 478, no. 7368, pp. 197-203, 2011.

[95] C. Genestet, A. le Gouellec, H. Chaker et al., "Scavenging of reactive oxygen species by tryptophan metabolites helps Pseudomonas aeruginosa escape neutrophil killing," Free Radical Biology \& Medicine, vol. 73, pp. 400-410, 2014.

[96] J. Reyes Ocampo, R. Lugo Huitrón, D. González-Esquivel et al., "Kynurenines with neuroactive and redox properties: relevance to aging and brain diseases," Oxidative Medicine and Cellular Longevity, vol. 2014, Article ID 646909, 22 pages, 2014.

[97] G. Giles, C. Collins, T. Stone, and C. Jacob, "Electrochemical and in vitro evaluation of the redox-properties of kynurenine species," Biochemical and Biophysical Research Communications, vol. 300, no. 3, pp. 719-724, 2003.

[98] L. Ramos-Chávez, R. Lugo Huitrón, D. González Esquivel et al., "Relevance of alternative routes of kynurenic acid production in the brain," Oxidative Medicine and Cellular Longevity, vol. 2018, Article ID 5272741, 14 pages, 2018.

[99] J. O'Connor, C. Andre, Y. Wang et al., "Interferon- and tumor necrosis Factor- Mediate the upregulation of indoleamine 2,3-dioxygenase and the induction of depressive-like behavior in mice in response to Bacillus Calmette-Guerin," Journal of Neuroscience, vol. 29, no. 13, pp. 4200-4209, 2009.

[100] Y. Huang, J. Ogbechi, F. Clanchy, R. Williams, and T. Stone, "IDO and kynurenine metabolites in peripheral and CNS disorders," Frontiers in Immunology, vol. 11, p. 388, 2020.

[101] A. Farzi, F. Reichmann, A. Meinitzer et al., "Synergistic effects of NOD1 or NOD2 and TLR4 activation on mouse sickness behavior in relation to immune and brain activity markers," Brain, Behavior, and Immunity, vol. 44, pp. 106-120, 2015.

[102] X. Zhu, Q. Tao, D. Sun-Waterhouse, W. Li, S. Liu, and C. Cui, " $\gamma$-[Glu]n-Trp ameliorates anxiety/depression-like behaviors and its anti-inflammatory effect in an animal model of anxiety/depression," Food \& Function, vol. 10, no. 9, pp. 55445554, 2019.

[103] B. Strasser, K. Becker, D. Fuchs, and J. Gostner, "Kynurenine pathway metabolism and immune activation: peripheral measurements in psychiatric and co-morbid conditions," Neuropharmacology, vol. 112, pp. 286-296, 2017.

[104] K. Bradley, J. Case, O. Khan et al., "The role of the kynurenine pathway in suicidality in adolescent major depressive disorder," Psychiatry Research, vol. 227, no. 2-3, pp. 206-212, 2015.

[105] D. Nation, M. Sweeney, A. Montagne et al., "Blood-brain barrier breakdown is an early biomarker of human cognitive dysfunction," Nature Medicine, vol. 25, no. 2, pp. 270-276, 2019.

[106] W. Abdullahi, D. Tripathi, and P. Ronaldson, "Blood-brain barrier dysfunction in ischemic stroke: targeting tight junctions and transporters for vascular protection," American Journal of Physiology-Cell Physiology, vol. 315, no. 3, pp. C343-C356, 2018.

[107] K. Jacobs, C. Lim, K. Blennow et al., "Correlation between plasma and CSF concentrations of kynurenine pathway metabolites in Alzheimer's disease and relationship to amyloid- $\beta$ and tau," Neurobiology of Aging, vol. 80, pp. 11-20, 2019.

[108] P. Chatterjee, H. Zetterberg, K. Goozee et al., "Plasma neurofilament light chain and amyloid- $\beta$ are associated with the kynurenine pathway metabolites in preclinical Alzheimer's disease," Journal of Neuroinflammation, vol. 16, no. 1, p. 186, 2019.

[109] L. Giil, Ø. Midttun, H. Refsum et al., "Kynurenine pathway metabolites in Alzheimer's disease," Journal of Alzheimer's Disease: JAD, vol. 60, no. 2, pp. 495-504, 2017.

[110] M. Mithaiwala, D. Santana-Coelho, G. Porter, and J. C. O'Connor, "Neuroinflammation and the kynurenine pathway in CNS disease: molecular mechanisms and therapeutic implications," Cells, vol. 10, no. 6, p. 1548, 2021.

[111] B. Lamas, J. Natividad, and H. Sokol, "Aryl hydrocarbon receptor and intestinal immunity," Mucosal Immunology, vol. 11, no. 4, pp. 1024-1038, 2018.

[112] T. Hubbard, I. Murray, and G. Perdew, "Indole and tryptophan metabolism: endogenous and dietary routes to Ah receptor activation," Drug Metabolism and Disposition: the biological fate of chemicals, vol. 43, no. 10, pp. 1522-1535, 2015.

[113] A. Stinn, J. Furkert, S. Kaufmann, P. Moura-Alves, and M. Kolbe, "Novel method for quantifying AhR-ligand binding affinities using microscale thermophoresis," Biosensors, vol. 11, no. 3, p. 60, 2021.

[114] S. Seok, Z. Ma, J. Feltenberger et al., "Trace derivatives of kynurenine potently activate the aryl hydrocarbon receptor 
(AHR)," The Journal of Biological Chemistry, vol. 293, no. 6, pp. 1994-2005, 2018.

[115] M. Cuartero, I. Ballesteros, J. de la Parra et al., "L-Kynurenine/aryl hydrocarbon receptor pathway mediates brain damage after experimental stroke," Circulation, vol. 130, no. 23, pp. 2040-2051, 2014.

[116] D. Kondrikov, A. Elmansi, R. Bragg et al., "Kynurenine inhibits autophagy and promotes senescence in aged bone marrow mesenchymal stem cells through the aryl hydrocarbon receptor pathway," Experimental Gerontology, vol. 130, article 110805, 2020.

[117] G. Manni, G. Mondanelli, G. Scalisi et al., "Pharmacologic induction of endotoxin tolerance in dendritic cells by Lkynurenine," Frontiers in Immunology, vol. 11, p. 292, 2020.

[118] Q. Li, J. Harden, C. Anderson, and N. Egilmez, "Tolerogenic phenotype of IFN- $\gamma$-induced IDO+Dendritic cells is maintained via an autocrine IDO-kynurenine/AhR-IDO loop," Journal of Immunology, vol. 197, no. 3, pp. 962-970, 2016.

[119] G. Mondanelli, A. Coletti, F. Greco et al., "Positive allosteric modulation of indoleamine 2,3-dioxygenase 1 restrains neuroinflammation," Proceedings of the National Academy of Sciences of the United States of America, vol. 117, no. 7, pp. 3848-3857, 2020.

[120] L. Du, Z. Xing, B. Tao et al., "Both IDO1 and TDO contribute to the malignancy of gliomas via the Kyn-AhR-AQP4 signaling pathway," Signal Transduction and Targeted Therapy, vol. 5, no. 1, p. 10, 2020.

[121] A. Colín-González, M. Maya-López, J. Pedraza-Chaverrí, S. Ali, A. Chavarría, and A. Santamaría, "The Janus faces of 3-hydroxykynurenine: dual redox modulatory activity and lack of neurotoxicity in the rat striatum," Brain Research, vol. 1589, pp. 1-14, 2014.

[122] A. Sardar, J. Bell, and G. Reynolds, "Increased concentrations of the neurotoxin 3-hydroxykynurenine in the frontal cortex of HIV-1-positive patients," Journal of Neurochemistry, vol. 64, no. 2, pp. 932-935, 1995.

[123] G. Leipnitz, C. Schumacher, K. Dalcin et al., "In vitro evidence for an antioxidant role of 3-hydroxykynurenine and 3-hydroxyanthranilic acid in the brain," Neurochemistry International, vol. 50, no. 1, pp. 83-94, 2007.

[124] C. Speciale and R. Schwarcz, "Uptake of kynurenine into rat brain slices," Journal of Neurochemistry, vol. 54, no. 1, pp. 156-163, 1990.

[125] J. Parrott, L. Redus, and J. C. O’Connor, "Kynurenine metabolic balance is disrupted in the hippocampus following peripheral lipopolysaccharide challenge," Journal of Neuroinflammation, vol. 13, no. 1, p. 124, 2016.

[126] P. Guidetti and R. Schwarcz, "3-Hydroxykynurenine potentiates quinolinate but not NMDA toxicity in the rat striatum," The European Journal of Neuroscience, vol. 11, no. 11, pp. 3857-3863, 1999.

[127] P. Song, T. Ramprasath, H. Wang, and M. Zou, “Abnormal kynurenine pathway of tryptophan catabolism in cardiovascular diseases," Cellular and Molecular Life Sciences: CMLS, vol. 74, no. 16, pp. 2899-2916, 2017.

[128] L. Darlington, C. Forrest, G. Mackay et al., "On the biological importance of the 3-hydroxyanthranilic acid: Anthranilic acid ratio," International Journal of Tryptophan Research: IJTR, vol. 3, article IJTR.S4282, 2010.

[129] D. Ramírez-Ortega, A. Ramiro-Salazar, D. González-Esquivel, C. Ríos, B. Pineda, and V. Pérez de la Cruz, "3-Hydroxy- kynurenine and 3-hydroxyanthranilic acid enhance the toxicity induced by copper in rat astrocyte culture," Oxidative Medicine and Cellular Longevity, vol. 2017, Article ID 2371895, 12 pages, 2017.

[130] G. Gadupudi and K. Chung, "Comparative genotoxicity of 3hydroxyanthranilic acid and anthranilic acid in the presence of a metal cofactor $\mathrm{Cu}$ (II) in vitro," Mutation Research, vol. 726, no. 2, pp. 200-208, 2011.

[131] T. Morita, K. Saito, M. Takemura et al., "3-Hydroxyanthranilic acid, an L-tryptophan metabolite, induces apoptosis in monocyte-derived cells stimulated by interferon- $\gamma$," Annals of Clinical Biochemistry, vol. 38, no. 3, pp. 242-251, 2001.

[132] D. Krause, H. Suh, L. Tarassishin et al., "The tryptophan metabolite 3-hydroxyanthranilic acid plays antiinflammatory and neuroprotective roles during inflammation: role of hemeoxygenase-1," The American Journal of Pathology, vol. 179, no. 3, pp. 1360-1372, 2011.

[133] M. Berg, K. Polyzos, H. Agardh et al., “3-Hydroxyanthralinic acid metabolism controls the hepatic SREBP/lipoprotein axis, inhibits inflammasome activation in macrophages, and decreases atherosclerosis in $\mathrm{Ldlr}^{-/}$mice," Cardiovascular Research, vol. 116, no. 12, pp. 1948-1957, 2020.

[134] J. Reyes-Ocampo, D. Ramírez-Ortega, G. I. Vázquez Cervantes et al., "Mitochondrial dysfunction related to cell damage induced by 3-hydroxykynurenine and 3-hydroxyanthranilic acid: non-dependent-effect of early reactive oxygen species production," Neurotoxicology, vol. 50, pp. 81-91, 2015.

[135] M. Gargaro, C. Vacca, S. Massari et al., "Engagement of nuclear coactivator 7 by 3-hydroxyanthranilic acid enhances activation of aryl hydrocarbon receptor in immunoregulatory dendritic cells," Frontiers in Immunology, vol. 10, p. 1973, 2019.

[136] C. Backhaus, H. Rahman, S. Scheffler, H. Laatsch, and R. Hardeland, "NO scavenging by 3-hydroxyanthranilic acid and 3-hydroxykynurenine: $N$-nitrosation leads via oxadiazoles to $o$-quinone diazides," Nitric Oxide: biology and chemistry, vol. 19, no. 3, pp. 237-244, 2008.

[137] M. P. Heyes, C. L. Achim, C. A. Wiley, E. O. Major, K. Saito, and S. P. Markey, "Human microglia convertl-tryptophan into the neurotoxin quinolinic acid," The Biochemical Journal, vol. 320, no. 2, pp. 595-597, 1996.

[138] F. Muller, W. Song, Y. Jang et al., "Denervation-induced skeletal muscle atrophy is associated with increased mitochondrial ROS production," American Journal of PhysiologyRegulatory, Integrative and Comparative Physiology, vol. 293, no. 3, pp. R1159-R1168, 2007.

[139] P. Pierozan, A. Zamoner, Â. Krombauer Soska et al., “Acute intrastriatal administration of quinolinic acid provokes hyperphosphorylation of cytoskeletal intermediate filament proteins in astrocytes and neurons of rats," Experimental Neurology, vol. 224, no. 1, pp. 188-196, 2010.

[140] C. Rios and A. Santamaria, "Quinolinic acid is a potent lipid peroxidant in rat brain homogenates," Neurochemical Research, vol. 16, no. 10, pp. 1139-1143, 1991.

[141] W. Behan, M. McDonald, L. Darlington, and T. Stone, "Oxidative stress as a mechanism for quinolinic acid-induced hippocampal damage: protection by melatonin and deprenyl," British Journal of Pharmacology, vol. 128, no. 8, pp. 17541760, 1999.

[142] F. Št'astný, V. Lisý, V. Mareš, V. Lisá, V. Balcar, and A. Santamaría, "Quinolinic acid induces NMDA receptor- 
mediated lipid peroxidation in rat brain microvessels," Redox Report, vol. 9, no. 4, pp. 229-233, 2004.

[143] T. Montine, M. Neely, J. Quinn et al., "Lipid peroxidation in aging brain and Alzheimer's disease1, 2," Free Radical Biology \& Medicine, vol. 33, no. 5, pp. 620-626, 2002.

[144] G. J. Guillemin, K. R. Williams, D. G. Smith, G. A. Smythe, J. Croitoru-Lamoury, and B. J. Brew, "Quinolinic acid in the pathogenesis of Alzheimer's disease," Advances in Experimental Medicine and Biology, vol. 527, pp. 167-176, 2003.

[145] M. Beal, R. Ferrante, K. Swartz, and N. Kowall, "Chronic quinolinic acid lesions in rats closely resemble Huntington's disease," The Journal of Neuroscience: the official journal of the Society for Neuroscience, vol. 11, no. 6, pp. 1649-1659, 1991.

[146] M. Heyes, R. Ellis, L. Ryan et al., "Elevated cerebrospinal fluid quinolinic acid levels are associated with region-specific cerebral volume loss in HIV infection," Brain: a journal of neurology, vol. 124, no. 5, pp. 1033-1042, 2001.

[147] P. Pierozan, F. Ferreira, B. de Lima, and R. Pessoa-Pureur, "Quinolinic acid induces disrupts cytoskeletal homeostasis in striatal neurons. Protective role of astrocyte-neuron interaction," Journal of Neuroscience Research, vol. 93, no. 2, pp. 268-284, 2015.

[148] A. Latif-Hernandez, D. Shah, T. Ahmed et al., "Quinolinic acid injection in mouse medial prefrontal cortex affects reversal learning abilities, cortical connectivity and hippocampal synaptic plasticity," Scientific Reports, vol. 6, no. 1, p. 36489, 2016.

[149] K. K. Ting, B. J. Brew, and G. J. Guillemin, "Effect of quinolinic acid on human astrocytes morphology and functions: implications in Alzheimer's disease," Journal of Neuroinflammation, vol. 6, no. 1, p. 36, 2009.

[150] E. Yan, T. Frugier, C. Lim et al., "Activation of the kynurenine pathway and increased production of the excitotoxin quinolinic acid following traumatic brain injury in humans," Journal of Neuroinflammation, vol. 12, no. 1, p. 110, 2015.

[151] G. Guillemin, S. Kerr, G. Smythe et al., "Kynurenine pathway metabolism in human astrocytes: a paradox for neuronal protection," Journal of Neurochemistry, vol. 78, no. 4, pp. 842853, 2001.

[152] C. Speciale, K. Hares, R. Schwarcz, and N. Brookes, "Highaffinity uptake of L-kynurenine by a $\mathrm{Na}^{+}$-independent transporter of neutral amino acids in astrocytes," The Journal of Neuroscience: the official journal of the Society for Neuroscience, vol. 9, no. 6, pp. 2066-2072, 1989.

[153] S. Beggiato, S. Tanganelli, K. Fuxe, T. Antonelli, R. Schwarcz, and L. Ferraro, "Endogenous kynurenic acid regulates extracellular GABA levels in the rat prefrontal cortex," Neuropharmacology, vol. 82, pp. 11-18, 2014.

[154] A. Zmarowski, H. Wu, J. Brooks et al., "Astrocyte-derived kynurenic acid modulates basal and evoked cortical acetylcholine release," The European Journal of Neuroscience, vol. 29, no. 3, pp. 529-538, 2009.

[155] E. Flores-Barrera, D. Thomases, D. Cass et al., "Preferential disruption of prefrontal GABAergic function by nanomolar concentrations of the $A 7 \mathrm{nACh}$ negative modulator kynurenic acid," The Journal of Neuroscience: the official journal of the Society for Neuroscience, vol. 37, no. 33, pp. 7921-7929, 2017.

[156] D. Ramirez Ortega, P. Ovalle Rodríguez, B. Pineda et al., "Kynurenine pathway as a new target of cognitive impairment induced by lead toxicity during the lactation," Scientific Reports, vol. 10, no. 1, p. 3184, 2020.
[157] J. Wang, N. Simonavicius, X. Wu et al., "Kynurenic Acid as a Ligand for Orphan G Protein-coupled Receptor GPR35," The Journal of Biological Chemistry, vol. 281, no. 31, pp. 2202122028, 2006.

[158] P. Pierozan, H. Biasibetti-Brendler, F. Schmitz, F. Ferreira, R. Pessoa-Pureur, and A. Wyse, "Kynurenic acid prevents cytoskeletal disorganization induced by quinolinic acid in mixed cultures of rat striatum," Molecular Neurobiology, vol. 55, no. 6, pp. 5111-5124, 2018.

[159] I. Lapin, “Antagonism of kynurenine-induced seizures by picolinic, kynurenic and xanthurenic Acids," Journal of Neural Transmission, vol. 56, no. 2-3, pp. 177-185, 1983.

[160] D. Bortz, H. Wu, R. Schwarcz, and J. Bruno, "Oral administration of a specific kynurenic acid synthesis (KAT II) inhibitor attenuates evoked glutamate release in rat prefrontal cortex," Neuropharmacology, vol. 121, pp. 69-78, 2017.

[161] A. Koshy Cherian, H. Gritton, D. Johnson, D. Young, R. Kozak, and M. Sarter, "A systemically-available kynurenine aminotransferase II (KAT II) inhibitor restores nicotine-evoked glutamatergic activity in the cortex of rats," Neuropharmacology, vol. 82, pp. 41-48, 2014.

[162] M. Potter, G. Elmer, R. Bergeron et al., "Reduction of endogenous kynurenic acid formation enhances extracellular glutamate, hippocampal plasticity, and cognitive behavior," Neuropsychopharmacology: official publication of the American College of Neuropsychopharmacology, vol. 35, no. 8, pp. 1734-1742, 2010.

[163] L. Peyton, A. Oliveros, M. Tufvesson-Alm et al., "Lipopolysaccharide increases cortical kynurenic acid and deficits in reference memory in mice," International Journal of Tryptophan Research: IJTR, vol. 12, 2019.

[164] L. Kubicova, F. Hadacek, G. Bachmann, W. Weckwerth, and V. Chobot, "Coordination complex formation and redox properties of kynurenic and xanthurenic acid can affect brain tissue homeodynamics," Antioxidants, vol. 8, no. 10, p. 476, 2019.

[165] F. Fazio, L. Lionetto, M. Curto et al., "Xanthurenic acid activates mGlu2/3 metabotropic glutamate receptors and is a potential trait marker for schizophrenia," Scientific Reports, vol. 5, p. 17799, 2015.

[166] F. Fazio, L. Lionetto, G. Molinaro et al., "Cinnabarinic acid, an endogenous metabolite of the kynurenine pathway, activates type 4 metabotropic glutamate receptors," Molecular Pharmacology, vol. 81, no. 5, pp. 643-656, 2012.

[167] M. Bosco, A. Rapisarda, S. Massazza, G. Melillo, H. Young, and L. Varesio, "The tryptophan catabolite picolinic acid selectively induces the chemokines macrophage inflammatory protein- $1 \alpha$ and $-1 \beta$ in macrophages," Journal of Immunology, vol. 164, no. 6, pp. 3283-3291, 2000.

[168] J. Prodinger, L. Loacker, R. Schmidt et al., “The tryptophan metabolite picolinic acid suppresses proliferation and metabolic activity of CD4+ T cells and inhibits c-Myc activation," Journal of Leukocyte Biology, vol. 99, no. 4, pp. 583-594, 2016.

[169] S. Cai, K. Sato, T. Shimizu et al., "Antimicrobial activity of picolinic acid against extracellular and intracellular Mycobacterium avium complex and its combined activity with clarithromycin, rifampicin and fluoroquinolones," The Journal of Antimicrobial Chemotherapy, vol. 57, no. 1, pp. 8593, 2006.

[170] Y. Ma, L. Yu, M. Olah et al., "Epigenomic features related to microglia are associated with attenuated effect ofAPOE $\varepsilon 4$ 
on Alzheimer's disease risk in humans," Alzheimer's \& Dementia, vol. 16, no. S2, 2021.

[171] the Alzheimer's Disease Neuroimaging Initiative, I. Katzourou, G. Leonenko et al., "Cognitive decline in Alzheimer's disease is not associated with APOE," Journal of Alzheimer's Disease: JAD, vol. 84, no. 1, pp. 141-149, 2021.

[172] F. de Leeuw, C. Peeters, M. Kester et al., "Blood-based metabolic signatures in Alzheimer's disease," Alzheimer's \& Dementia: Diagnosis, Assessment \& Disease Monitoring, vol. 8, no. 1, pp. 196-207, 2017.

[173] G. Paglia, M. Stocchero, S. Cacciatore et al., "Unbiased metabolomic investigation of Alzheimer's disease brain points to dysregulation of mitochondrial aspartate metabolism," Journal of Proteome Research, vol. 15, no. 2, pp. 608-618, 2016.

[174] J. Tynkkynen, V. Chouraki, S. van der Lee et al., “Association of branched-chain amino acids and other circulating metabolites with risk of incident dementia and Alzheimer's disease: a prospective study in eight cohorts," Alzheimer's \& Dementia, vol. 14, no. 6, pp. 723-733, 2018.

[175] J. Toledo, M. Arnold, G. Kastenmüller et al., "Metabolic network failures in Alzheimer's disease: a biochemical road map," Alzheimer's \& Dementia, vol. 13, no. 9, pp. 965-984, 2017.

[176] V. Varma, A. Oommen, S. Varma et al., "Brain and blood metabolite signatures of pathology and progression in Alzheimer disease: a targeted metabolomics study," PLoS Medicine, vol. 15, no. 1, article e1002482, 2018.

[177] Y. Xin, L. Zhang, J. Hu, H. Gao, and B. Zhang, "Correlation of early cognitive dysfunction with inflammatory factors and metabolic indicators in patients with Alzheimer's disease," American Journal of Translational Research, vol. 13, pp. 9208-9215, 2021.

[178] C. Jack, D. Bennett, K. Blennow et al., "NIA-AA research framework: toward a biological definition of Alzheimer's disease," Alzheimer's \& Dementia, vol. 14, no. 4, pp. 535-562, 2018.

[179] on behalf of AddNeuroMed consortium, L. Whiley, K. E. Chappell et al., "Metabolic phenotyping reveals a reduction in the bioavailability of serotonin and kynurenine pathway metabolites in both the urine and serum of individuals living with Alzheimer's disease," Alzheimer's Research \& Therapy, vol. 13, no. 1, p. 20, 2021.

[180] M. González-Sánchez, J. Jiménez, A. Narváez et al., “Kynurenic acid levels are increased in the CSF of Alzheimer's disease patients," Biomolecules, vol. 10, no. 4, p. 571, 2020.

[181] A. Willette, C. Pappas, N. Hoth et al., "Inflammation, negative affect, and amyloid burden in Alzheimer's disease: insights from the kynurenine pathway," Brain, Behavior, and Immunity, vol. 95, pp. 216-225, 2021.

[182] E. Gulaj, K. Pawlak, B. Bien, and D. Pawlak, "Kynurenine and its metabolites in Alzheimer's disease patients," Advances in Medical Sciences, vol. 55, no. 2, pp. 204-211, 2010.

[183] N. Braidy and R. Grant, "Kynurenine pathway metabolism and neuroinflammatory disease," Neural Regeneration Research, vol. 12, no. 1, pp. 39-42, 2017.

[184] F. Sorgdrager, Y. Vermeiren, M. Van Faassen et al., "Age- and disease-specific changes of the kynurenine pathway in Parkinson's and Alzheimer's disease," Journal of Neurochemistry, vol. 151, no. 5, pp. 656-668, 2019.

[185] L. Tan, J. Yu, and L. Tan, "The kynurenine pathway in neurodegenerative diseases: mechanistic and therapeutic consider- ations," Journal of the Neurological Sciences, vol. 323, no. 1-2, pp. 1-8, 2012.

[186] G. Keilhoff and G. Wolf, "Memantine prevents quinolinic acid-induced hippocampal damage," European Journal of Pharmacology, vol. 219, no. 3-4, pp. 451-454, 1992.

[187] Z. Kincses, J. Toldi, and L. Vécsei, "Kynurenines, neurodegeneration and Alzheimer's disease," Journal of Cellular and Molecular Medicine, vol. 14, no. 8, pp. 2045-2054, 2010.

[188] H. Wu, S. Lee, and R. Schwarcz, "Systemic administration of 4-chlorokynurenine prevents quinolinate neurotoxicity in the rat hippocampus," European Journal of Pharmacology, vol. 390, no. 3, pp. 267-274, 2000.

[189] C. Parli, P. Krieter, and B. Schmidt, "Metabolism of 6chlorotryptophan to 4-chloro-3-hydroxyanthranilic acid: a potent inhibitor of 3-hydroxyanthranilic acid oxidase," Archives of Biochemistry and Biophysics, vol. 203, no. 1, pp. 161-166, 1980.

[190] I. Plangár, D. Zádori, P. Klivényi, J. Toldi, and L. Vécsei, "Targeting the kynurenine pathway-related alterations in Alzheimer's disease: a future therapeutic strategy," Journal of Alzheimer's Disease: JAD, vol. 24, no. s2, pp. 199-209, 2011.

[191] E. Vamos, A. Pardutz, P. Klivenyi, J. Toldi, and L. Vecsei, "The role of kynurenines in disorders of the central nervous system: possibilities for neuroprotection," Journal of the Neurological Sciences, vol. 283, no. 1-2, pp. 21-27, 2009.

[192] D. Zwilling, S. Huang, K. Sathyasaikumar et al., "Kynurenine 3-monooxygenase inhibition in blood ameliorates neurodegeneration," Cell, vol. 145, no. 6, pp. 863-874, 2011.

[193] G. Deora, S. Kantham, S. Chan et al., "Multifunctional analogs of kynurenic acid for the treatment of Alzheimer's disease: synthesis, pharmacology, and molecular modeling studies," ACS Chemical Neuroscience, vol. 8, no. 12, pp. 2667-2675, 2017.

[194] D. Yu, B. Tao, Y. Yang et al., "The IDO inhibitor coptisine ameliorates cognitive impairment in a mouse model of Alzheimer's disease," Journal of Alzheimer's Disease: JAD, vol. 43, pp. 291-302, 2014.

[195] E. Fertan, K. Stover, M. Brant et al., "Effects of the novel IDO inhibitor DWG-1036 on the behavior of male and female 3xTg-AD mice," Frontiers in Pharmacology, vol. 10, p. 1044, 2019.

[196] C. Breda, K. Sathyasaikumar, S. Sograte Idrissi et al., "Tryptophan-2,3-dioxygenase (TDO) inhibition ameliorates neurodegeneration by modulation of kynurenine pathway metabolites," Proceedings of the National Academy of Sciences of the United States of America, vol. 113, no. 19, pp. 54355440, 2016.

[197] W. Wu, J. Nicolazzo, L. Wen et al., "Expression of tryptophan 2,3-dioxygenase and production of kynurenine pathway metabolites in triple transgenic mice and human Alzheimer's disease brain," PloS One, vol. 8, no. 4, article e59749, 2013.

[198] N. Woodling, D. Colas, Q. Wang et al., "Cyclooxygenase inhibition targets neurons to prevent early behavioural decline in Alzheimer's disease model mice," Brain: a journal of neurology, vol. 139, no. 7, pp. 2063-2081, 2016.

[199] G. Zakhary, P. Sherchan, Q. Li, J. Tang, and J. Zhang, “Modification of kynurenine pathway via inhibition of kynurenine hydroxylase attenuates surgical brain injury complications in a male rat model," Journal of Neuroscience Research, vol. 98, pp. 155-167, 2019. 